\title{
Overexpression of aquaporin 2 in renal tubular epithelial cells alleviates pyroptosis
}

\author{
Yu Fan ${ }^{1,2,3 \#}$, Ming Ma ${ }^{1,2 \#}$, Xiaobing Feng ${ }^{1,2}$, Turun Song ${ }^{1,2}$, Qiang Wei ${ }^{1,3}$, Tao Lin ${ }^{1,2,3}$ \\ ${ }^{1}$ Urology Department, Urology Research Institute, West China Hospital, Sichuan University, Chengdu, China; ${ }^{2}$ Organ Transplantation Center, West \\ China Hospital, Sichuan University, Chengdu, China; ${ }^{3}$ National Clinical Research Center for Geriatrics, West China Hospital, Sichuan University, \\ Chengdu, China \\ Contributions: (I) Conception and design: T Lin; (II) Administrative support: Q Wei, T Lin; (III) Provision of study materials or patients: Y Fan, \\ T Song, M Ma; (IV) Collection and assembly of data: M Ma, Y Fan, X Feng; (V) Data analysis and interpretation: M Ma, Y Fan; (VI) Manuscript \\ writing: All authors; (VII) Final approval of manuscript: All authors. \\ \#These authors contributed equally to this work. \\ Correspondence to: Tao Lin. Department of Urology/Institute of Urology, West China Hospital, Sichuan University, 37\# Guoxue Alley, Chengdu \\ 610041, China. Email: kidney5@163.com.
}

\begin{abstract}
Background: Severe renal ischemia-reperfusion injury results in worse outcomes of kidney transplantation. Compared to the collecting duct, the proximal tubule is more likely to exhibit severe pyroptosis and damage during renal ischemia-reperfusion. Aquaporins were reported of having regulatory roles in pyroptosis. We explored whether aquaporin 2 overexpression in proximal tubular cells could alleviate ischemia-reperfusion injury related pyroptosis.
\end{abstract}

Methods: A renal ischemia-reperfusion model of mice was established, and human kidney 2 cells were treated with hypoxia-reoxygenation. Aquaporin 2 overexpression was achieved in human kidney 2 cells transfected with lentivirus, which were then cultured with murine cells. Renal tissues and serum of the mice, and human kidney 2 cells were subjected to histological, molecular, and biochemical examinations.

Results: Compared with the sham group, the renal function of the ischemia-reperfusion group was significantly decreased, and the tissue injury was severe and accompanied by more nuclear dissolved and necrosis. Besides, the expression of aquaporin 1-5 decreased significantly, while the expression of Toll-like receptor 4, caspase-1, kim-1 and interleukin $1 \beta$ and 18 increased significantly in ischemia-reperfusion group. Similar results were observed in the human kidney 2 cells test. Overexpression of aquaporin 2 partially reversed the cell damage, pyroptosis, and molecular expression changes of human kidney 2 cells induced by hypoxia-reoxygenation.

Conclusions: Our findings suggest that aquaporin 2 overexpression can potentially reduce pyroptosis in proximal tubular cells, and thus might be a novel target for relieving pyroptosis and injury in renal ischemiareperfusion injury.

Keywords: Aquaporin; ischemia-reperfusion injury; kidney transplantation; pyroptosis

Submitted Jan 24, 2021. Accepted for publication Apr 21, 2021.

doi: $10.21037 /$ tau-21-71

View this article at: http://dx.doi.org/10.21037/tau-21-71

\section{Introduction}

Kidney transplantation is the optimal treatment for endstage renal disease and is far superior to hemodialysis in terms of long-term survival rate, quality of life and treatment cost (1). In most cases, allografts undergo warm and cold ischemia and subsequent ischemia-reperfusion injury (IRI). Allografts with longer ischemic time (14 hours) were reported to be more likely to demonstrate delayed graft function, and the risk of graft loss increased by $1 \%$ for per hour increase in the duration of total ischemia in 
comparison with allografts with shorter ischemic time $(<14$ hours) (2). As a result of necrosis of renal tubular epithelial cells, renal IRI not only delays the recovery of graft function, but also promotes rejection. Because of specific immunity that results from antigen exposure caused by injury, renal IRI affects the long-term survival rate of renal allografts and recipients (3). Evidence indicating that allografts with early resistance to IRI have significantly enhanced function 5 years after transplantation in recipients and may prolong life is robust (4). Although good results have been achieved, the alleviation of IRI and the improvement in prognosis are still not ideal (5).

During IRI, in comparison with the collecting duct, the renal proximal tubule is more susceptible to tissue damage that is more likely to be severe. Phenotypic differences between the collecting duct and the proximal tubule might account for the difference in susceptibility. Aquaporin 2 (AQP2), one member of aquaporin (AQP) family, is considered to be expressed mostly in the collecting duct, and its regulating action is responsible for fine-tuning the final urine volume to meet the current demands of the body (6). Besides being involved in fluid transport, research has revealed the modulatory role of AQPs in interleukin (IL)-1 $\beta$-related inflammation (including pyroptosis), in pathophysiological processes in tissue and organ inflammation, in oxidative stress and in IRI (7). AQP deletion is linked to worse prognosis of patients with renal IRI (8). Moreover, the regulating activities of AQPs can affect the survival of allografts (7). However, there is no evidence clarifying the effect of AQPs on pyroptosis in renal IRI.

Because of the differences between the collecting duct and the proximal tubule in response to IRI and their differential expressions of AQPs, we evaluated the potential effects of AQPs on renal IRI, with a particular focus on the AQP2 and the process of pyroptosis.

We present the following article in accordance with the ARRIVE reporting checklist (available at http://dx.doi. org/10.21037/tau-21-71).

\section{Methods}

\section{The renal IRI model in mice}

Adult C57BL/6 male mice (eight weeks old; Chengdu Dossy Experimental Animals, Co., Ltd., Chengdu, China) were raised under standard guidelines. The Animal Ethics Committee of West China Hospital, Sichuan University, Chengdu, China (No. 2019141A) approved this study. All experimental procedures were followed by the National Institutes of Health Guide for the Care and Use of Laboratory Animals. Fifteen mice were fed adaptively for 7 days and then randomly divided into two groups. To establish a renal IRI model, the bilateral renal pedicles were clamped simultaneously with a non-invasive artery clamp for 30 minutes (Figure S1, $\mathrm{n}=8$ ); for the sham model, the operation lasted for 30 minutes without clamping $(n=7)$. All mice were sacrificed after 24 hours, and their blood and renal tissues were collected.

\section{The buman kidney 2 (HK-2) cell hypoxia-reoxygenation (H/R) model}

Both HK-2 and 293T cells were obtained from the Institute of Urology, West China Hospital of Sichuan University, Chengdu, China. HK-2 cells were cultured according to the recommended methods of the American Type Culture Collection (9). Some cells were cultured in conditions of hypoxia for 24 hours and then in normal oxygen conditions for 12 hours to achieve H/R, as previously described (10).

A lentiviral expression vector (pLVX-AcGFP1-N1) was utilized to construct plasmids containing the target gene AQP2 (NCBI Reference Sequence: NM_000486.5). The lentivirus was packaged and concentrated through 293 T cell transfection. The virus titre was measured under a fluorescence microscope (Figure S2). HK-2 cells were transfected to obtain cells that overexpressed AQP2 (AQP2hi cells) and control cells created through the use of non-related blank vectors (NR cells).

HK-2 cells were added to $6-\left(3 \times 10^{5}\right.$ cells per well $), 24-$ $\left(2.5 \times 10^{4}\right.$ cells per well), or 96 -well plates $\left(4 \times 10^{3}\right.$ cells per well) and cultured with or without H/R. Thereafter, cells were divided into the following six groups: negative control (NC, cells cultured without $\mathrm{H} / \mathrm{R}$ and transfection), H/R (cells cultured with $\mathrm{H} / \mathrm{R}$, but without transfection), NR (NR cells cultured without $\mathrm{H} / \mathrm{R}$ ), AQP2hi (AQP2hi cells cultured without $H / R), N R+H / R$ (NR cells cultured with $H / R$ ), and AQP2hi + H/R (AQP2hi cells cultured with H/R).

\section{Histopathological staining}

The collected renal tissues of the mice were formalinfixed, paraffin-embedded and then sectioned for subsequent haematoxylin-eosin (HE) staining. The following procedures were performed as previously described.

Immunofluorescence staining was performed to assess the expression of AQP2 and caspase-1. For frozen-section immunofluorescence staining, sections were fixed, blocked 
and then stained with the primary antibodies AQP2 (1:500, PAA580Hu01; Cloud-Clone Corp., Wuhan, China) and caspase-1 (1:100, PAB592Hu01; Cloud-Clone Corp.). The fluorescent secondary antibodies were fluorescein isothiocyanate-labeled goat anti-rabbit immunoglobulin G (1:500, A0562; Beyotime Biotechnology Co., Ltd., Haimen, Jiangsu, China) and 4',6-diamidino-2-phenylindole (C1002; Beyotime Biotechnology Co., Ltd.). Sections were then mounted with anti-fade mounting medium (Abcam Co., Ltd, Cambridge, MA, USA).

FAM-FLICA kits (ICT098; ImmunoChemistry Technologies LLC., Bloomington, MN, USA) were utilized to conduct FAM-YVAD-FMK/Hoechst/propidium iodide (PI) staining according to the manufacturer's instructions. Images were collected using a fluorescence microscope.

\section{Cell proliferation assay}

Cell proliferation was assessed with the Cell Counting Kit8 (CCK8; C0037; Beyotime Biotechnology Co., Ltd.). Cells were added to 96 -well plate $\left(5 \times 10^{3}\right.$ cells per well) and cultured as described above. Then, reagent containing $10 \%$ CCK8 solution was added, and plates were incubated for 4 hours. Optical density (OD) values for calculating cell proliferation were measured at $450 \mathrm{~nm}$ with using a microplate reader (EnVision, PerkinElmer Co., Ltd., Shanghai, China).

The concentration lactate dehydrogenase $(L D H), I L-1 \beta$, $I L-18$, serum creatinine (Scr), blood urea nitrogen (BUN), and caspase-1 activity

The release rates of LDH (C0016; Beyotime Biotechnology Co., Ltd.), Scr (C011-2; Nanjing Jiancheng Bioengineering Institute, Nanjing, Jiangsu, China) and BUN (C0132; Nanjing Jiancheng Bioengineering Institute) were measured with corresponding test kits; IL-1 $\beta$ (SEA563Mu; Wuhan USCN Business Co., Ltd., Wuhan, China) and IL-18 (SEA064Mu; Wuhan USCN Business Co., Ltd.) were measured with enzyme-linked immunosorbent assay kits; and caspase- 1 activity was measured with the Caspase 1 Activity Assay kit (C1101; Beyotime Biotechnology Co., Ltd.). All tests were performed according to the manufacturer's instructions.

\section{Quantitative real-time polymerase chain reaction (qRT-PCR)}

RNAs of tissues/cells were extracted with TRIzol; complementary DNAs were synthesized with the iScript cDNA Synthesis Kit (Bio-Rad Laboratories, Hercules, CA, USA). After reverse transcription and dilution, primers (see Appendix 1 for details) were applied to PCR reactions.

\section{Western blotting}

Radioimmunoprecipitation assay lysate solution was used to prepare cell/tissue lysates. Protein concentrations were measured with the BCA Protein Assay Kit (Beyotime Biotechnology Co. Ltd, Haimen, Jiangsu, China). After membrane transfer and blocking, primary antibodies, including caspase-1, IL-1 $\beta$, AQP2, kidney injury molecule 1 (kim-1) and glyceraldehyde 3-phosphate dehydrogenase (GAPDH), were incubated. Then, the corresponding secondary antibodies were incubated, and gels were detected with chemiluminescence.

\section{Statistical analysis}

The data were calculated as means \pm standard deviations. All analyses were performed with SPSS version 22.0 (SPSS Inc., Chicago, IL, USA) and GraphPad Prism version 7.0 (GraphPad Software Inc., La Jolla, CA, USA). Among multiple groups, the one-way NOVA analysis, followed with a Tukey test was used to compare the differences of all column pairs. For difference between two groups, Student's $t$-test was applied. $\mathrm{P}<0.05$ was considered to be significant.

\section{Results}

\section{Renal IRI resulted in pyroptosis and downregulated the expression of aquaporins}

In renal tissues of mice with IRI $(\mathrm{n}=8)$, severe exfoliation of renal tubular epithelial cells was observed, the nuclei of which were dissolved and necrotic. Some renal tubules were dilated and ruptured (Figure 1). These renal tissues had significantly less relative messenger RNA (mRNA) expressions of AQP1-AQP5 (all $\mathrm{P}<0.05$; Figure 1), and less protein expression of $\mathrm{AQP} 2$ than did the renal tissues from the sham group $(\mathrm{P}=0.019 ;$ Figure $1 ; \mathrm{n}=7)$, and on the immunofluorescence test $(\mathrm{P}=0.031$; Figure 2). However, tissues from the IRI group ( $\mathrm{n}=8)$, compared with those from the sham group $(n=7)$, exhibited significantly more mRNA expression of Toll-like receptor 4 (Figure 1); higher activity of caspase-1 $(\mathrm{P}<0.001$; Figure 1$)$; and more protein expression of kim-1 ( $\mathrm{P}=0.010$; Figure 1$), \mathrm{IL}-1 \beta(\mathrm{P}=0.015$; 

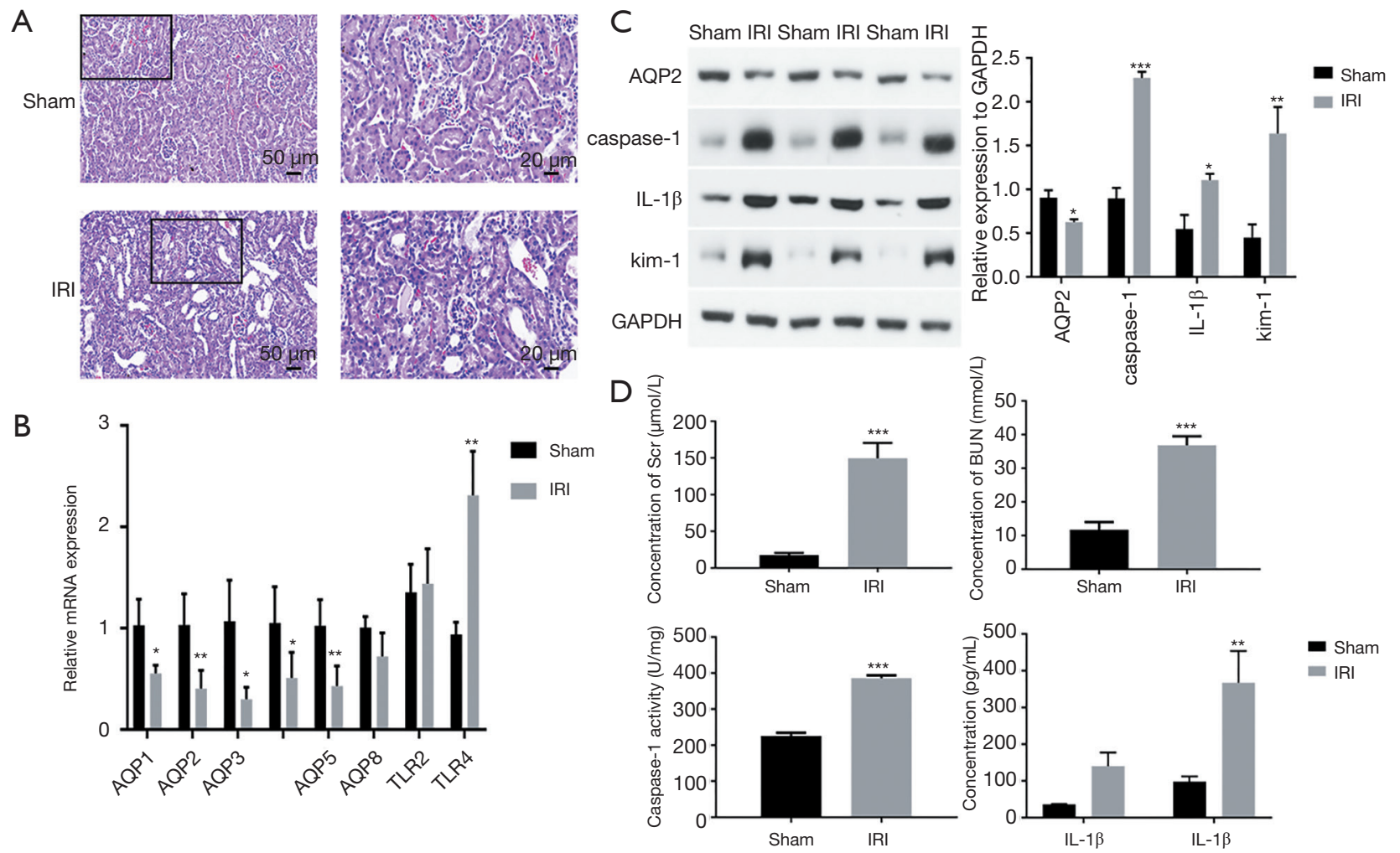

Figure 1 Renal IRI resulted in pyroptosis and downregulation of AQPs in vivo. (A) HE staining. In the renal tissues of the IRI group (n=8), severe exfoliation of renal tubular epithelial cells was observed, of which the nuclei were dissolved and necrotic. Some renal tubules were dilated and ruptured. (B) qRT-PCR analysis. IRI renal tissues had significantly less mRNA expressions of AQP1-AQP5 and numerically less mRNA expression of AQP8 but significantly more relative mRNA expression of Toll-like receptor 4. (C) Western blot analysis. IRI renal tissues had significantly less expression of AQP2 but significantly more expression of kim-1, IL- $1 \beta$ and caspase-1 than renal tissues of the sham group (n=7). (D) Measurement of serum concentration of IL-1 $\beta$, IL-18, Scr and BUN, and renal activity of caspase-1 test. IRI group had higher serum concentrations of Scr, BUN, IL-1 $\beta$ and IL-18, and higher activity of caspase-1 than the sham group. Compared with the sham group: *means $0.01 \leq \mathrm{P} \leq 0.05$; ${ }^{* *}$ means $0.001 \leq \mathrm{P} \leq 0.01$; ${ }^{* * *}$ means $\mathrm{P}<0.001$. AQP, aquaporin; BUN, blood urea nitrogen; HE, haematoxylin-eosin; IL, interleukin; IRI, ischemia-reperfusion injury; kim-1, kidney injury molecule 1; mRNA, messenger RNA; Scr, serum creatinine.

Figure 1) and caspase-1 (on Western blot test, $\mathrm{P}<0.001$, Figure 1; and on immunofluorescence test, $\mathrm{P}<0.001$, Figure 2). In addition, the IRI group, in comparison with the sham group, exhibited higher serum concentrations of Scr $(\mathrm{P}<0.001), \mathrm{BUN}(\mathrm{P}<0.001), \mathrm{IL}-1 \beta(\mathrm{P}=0.003)$ and IL-18 $(\mathrm{P}=0.002$; Figure 1$)$.

\section{H/R promoted pyroptosis and downregulated AQP2 in HK-2 cells}

In the CCK8 test (Figure 3), OD values were all significantly lower in the H/R group than that in the NC group after 24-, 36-, 48-, and 60-hour cultures (all $\mathrm{P}<0.01$ ) at the same time point. The H/R group, in comparison with the NC group, demonstrated significantly less expression of AQP2 by mRNA ( $\mathrm{P}=0.019$, Figure 3$)$ and protein $(\mathrm{P}=0.008$, Figure 3$)$, while the H/R group had significantly higher activity of caspase- $1(\mathrm{P}<0.001$, Figure 3$)$ and more expression of kim-1 $(\mathrm{P}<0.001$, Figure 3), IL-1 $\beta(\mathrm{P}<0.001$, Figure 3$)$ and caspase- 1 $(\mathrm{P}=0.012$, Figure 3$)$. In the supernatant of the H/R group, in comparison with the control group, concentrations of IL-1 $\beta(\mathrm{P}=0.023)$ and IL-18 $(\mathrm{P}=0.012)$ were higher, as well as the release rate of LDH (Figure 3). According to FAM-YVAD-FMK/Hoechst/PI staining, the fluorescence intensity of both FAM-YVAD-FMK and PI in the H/R group was higher than that in the control group (Figure 4). 

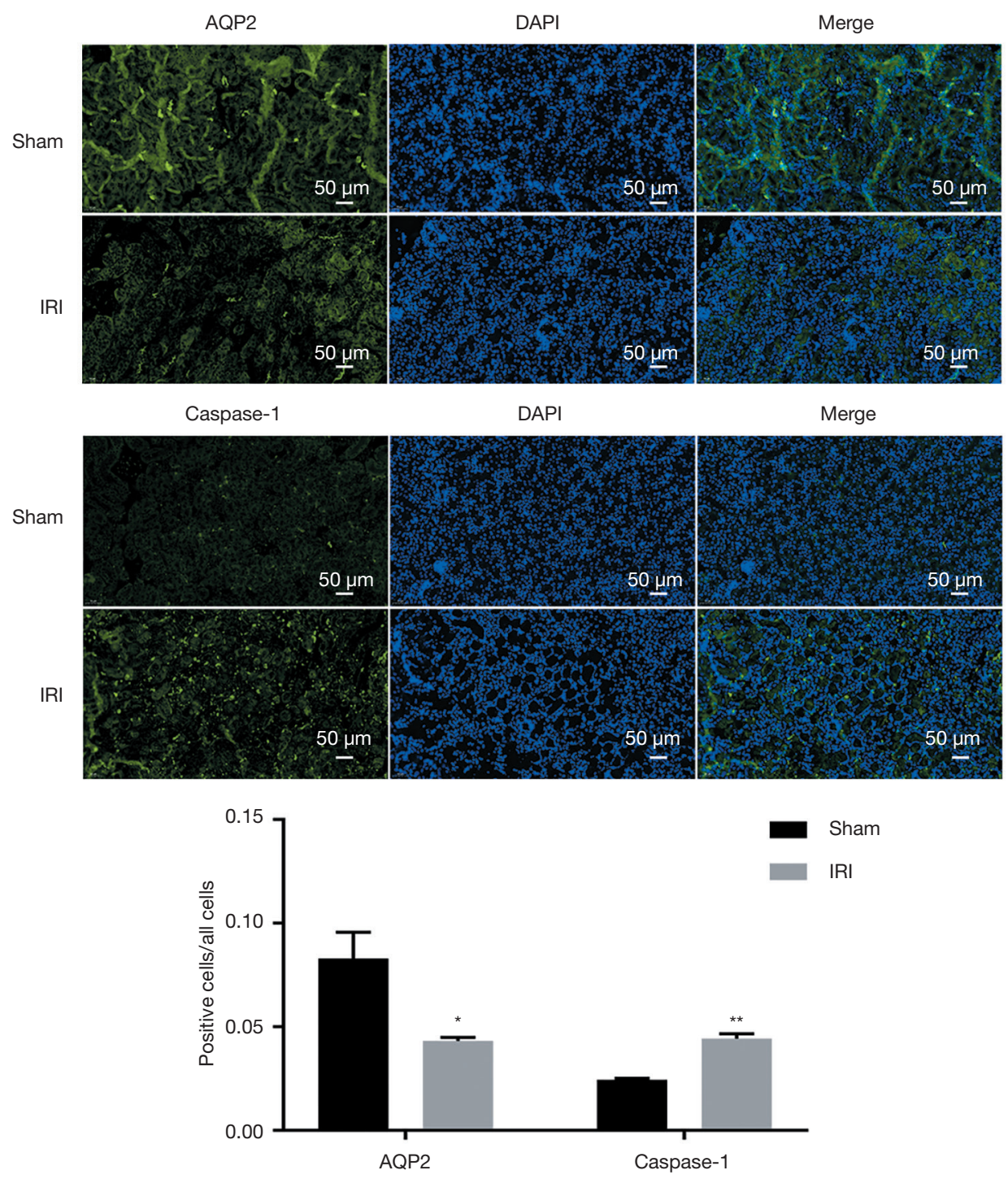

Figure 2 Immunofluorescence staining of renal tissues. Tissues from mice with IRI $(n=8)$ demonstrated significantly lower fluorescence intensity of AQP2 but higher fluorescence intensity of caspase-1 than those from the mice that underwent sham surgery (n=7). AQP, aquaporin; IRI, ischemia-reperfusion injury. * means $0.01 \leq \mathrm{P} \leq 0.05 ;{ }^{* *}$ means $0.001 \leq \mathrm{P} \leq 0.01$.

\section{Upregulation of AQP 2 alleviated H/R induced pyroptosis of HK-2 cells}

The AQP2 vector achieved acceptable overexpression efficiency (Figure 5; supplementary materials showed details). In the CCK8 test (Figure 5), OD values were all significantly lower in the NR $+\mathrm{H} / \mathrm{R}$ group than in the NR and AQP2hi + H/R group after 24-, 36-, 48-, and 60-hour culture (all $\mathrm{P}<0.05)$ at the same time point. In contrast, after 24-, 36-, 48-, and 60-hour culture, OD values were higher in the AQP2hi group than in the AQP2hi + H/R group (all $\mathrm{P}<0.05)$ and were comparable with those in the NR group (all $\mathrm{P}>0.05$ ). In the Western blot assay (Figure 5), the NR $+\mathrm{H} / \mathrm{R}$ group, in comparison with the NR group, exhibited significantly less expression of AQP2 $(\mathrm{P}=0.014)$ but more expression of kim-1 $(\mathrm{P}<0.001), \mathrm{IL}-1 \beta(\mathrm{P}=0.010)$ and caspase-1 $(\mathrm{P}=0.003)$. Similarly, the AQP2hi $+\mathrm{H} / \mathrm{R}$ group, in comparison with the AQP2hi group, expressed less AQP2 $(\mathrm{P}=0.009)$ but more kim-1 ( $\mathrm{P}=0.012), \mathrm{IL}-1 \beta(\mathrm{P}=0.006)$ and 

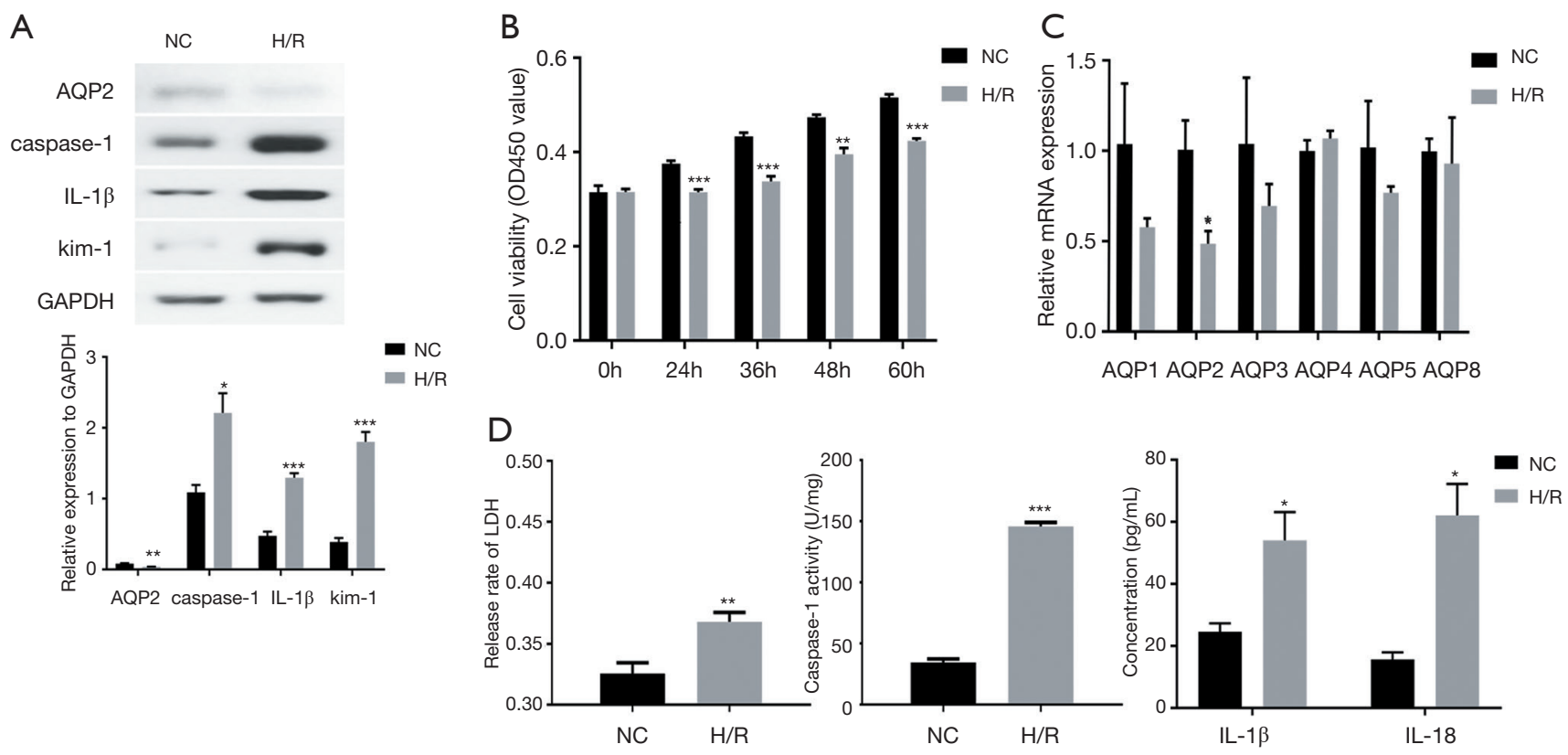

Figure $3 \mathrm{H} / \mathrm{R}$ resulted in pyroptosis and downregulation of AQP2 in HK-2 cells. (A) Western blot analysis. Tissues from mice that received H/R (the H/R group) expressed significantly less AQP2 but significantly more kim-1, IL-1 $\beta$ and caspase-1 than did those of the NC group. (B) Testing with the Cell Counting Kit-8. The OD values at the same time point were all significantly lower in the H/R group than in the NC group after 24-, 36-, 48-, and 60-hour culture. (C) qRT-PCR analysis. In the H/R group, mRNA expressed significantly less AQP2 and numerically less AQP1, APQ3 and AQP5 than did the NC group; expressions of AQP4 and AQP8 were comparable between the two groups. (D) Measurement of concentration of IL-1 $\beta$ and IL-18 in supernatant, and release rate of LDH and activity of caspase-1 in cells. Concentrations of IL-1 $\beta$ and IL-18 in the supernatant were significantly higher for the H/R group than for the NC group. The release rate of LDH and activity of caspase-1 was significantly higher in the H/R group than in the NC group. Compared with the sham group: *means $0.01 \leq \mathrm{P} \leq 0.05$; ** means $0.001 \leq \mathrm{P} \leq 0.01$; ${ }^{* * *}$ means $\mathrm{P}<0.001$. AQP, aquaporin; HK-2, human kidney 2; H/R, hypoxia-reoxygenation; IL, interleukin; IRI, ischemia-reperfusion injury; kim-1, kidney injury molecule 1; LDH, lactate dehydrogenase; mRNA, messenger RNA; NC, negative control; OD, optical density.

caspase-1 $(\mathrm{P}=0.002)$. However, the AQP2hi $+\mathrm{H} / \mathrm{R}$ group, in comparison with the $\mathrm{NR}+\mathrm{H} / \mathrm{R}$ group, expressed less kim-1 $(\mathrm{P}=0.002), \mathrm{IL}-1 \beta(\mathrm{P}=0.049)$ and caspase-1 $(\mathrm{P}=0.017)$.

The AQP2hi + H/R group had lower caspase-1 activity than the $\mathrm{NR}+\mathrm{H} / \mathrm{R}$ group $(\mathrm{P}=0.002$, Figure 5). The concentrations of IL- $1 \beta$, IL-18, and the release rate of LDH were higher in the supernatant of the $\mathrm{NR}+\mathrm{H} / \mathrm{R}$ group than that of the NR group and higher in the AQP2hi $+\mathrm{H} / \mathrm{R}$ group than in the AQP2hi group (all $\mathrm{P}<0.05$ ). The supernatant of the AQP2hi $+\mathrm{H} / \mathrm{R}$ group had a significantly lower concentration of IL-18 $(\mathrm{P}=0.024)$ and release rate of $\mathrm{LDH}(\mathrm{P}=0.004)$ and a non-significantly lower concentration of IL-1 $\beta(\mathrm{P}=0.065)$ than $\mathrm{did}$ the $\mathrm{NR}+\mathrm{H} / \mathrm{R}$ group. In FAM-YVAD-FMK/Hoechst/PI staining, the fluorescence intensities of both FAM-YVAD-FMK and PI were higher in the $\mathrm{NR}+\mathrm{H} / \mathrm{R}$ and $\mathrm{AQP} 2 \mathrm{hi}+\mathrm{H} / \mathrm{R}$ groups than in the NR and AQP2hi groups (Figure 4). The AQP2hi + H/R group showed weaker FAM-YVAD-FMK and PI fluorescence intensities than did the $\mathrm{NR}+\mathrm{H} / \mathrm{R}$ group.

\section{Discussion}

Previous study has reported that the urinary exosomal release of $A Q P 1$ and $A Q P 2$ during severe acute kidney injury in mice were significant decreased (11). The expression of renal AQP1 and AQP2 were also reported to be downregulated when urine volume was decreased in recipients of kidney transplants, and AQP2 downregulation was related to a decline in the glomerular filtration rate (12). In our study, we found the mRNA expression of AQP1AQP5 were significantly decreased and the AQP8 was numerically decreased after IRI initiation in mice kidneys. 

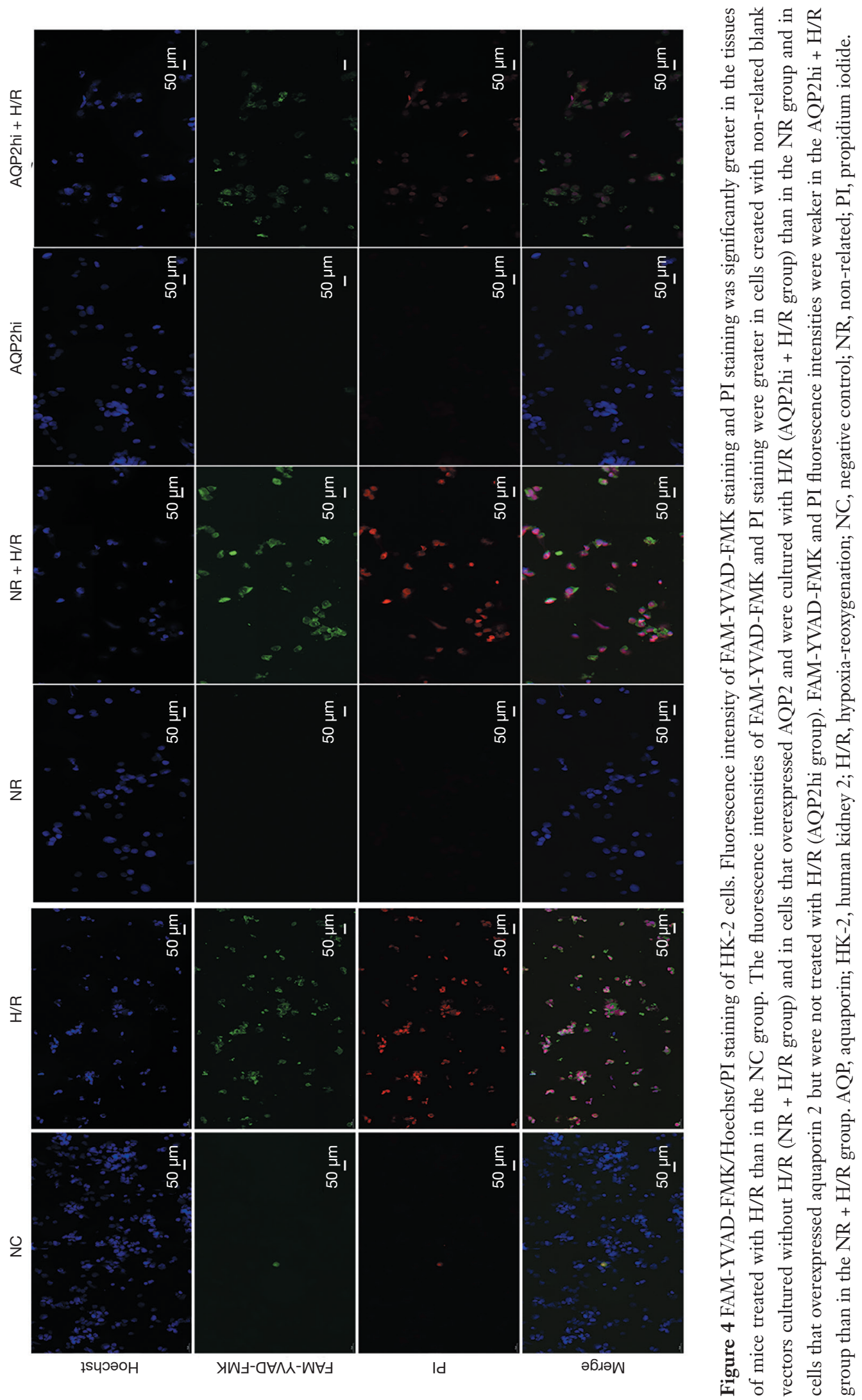

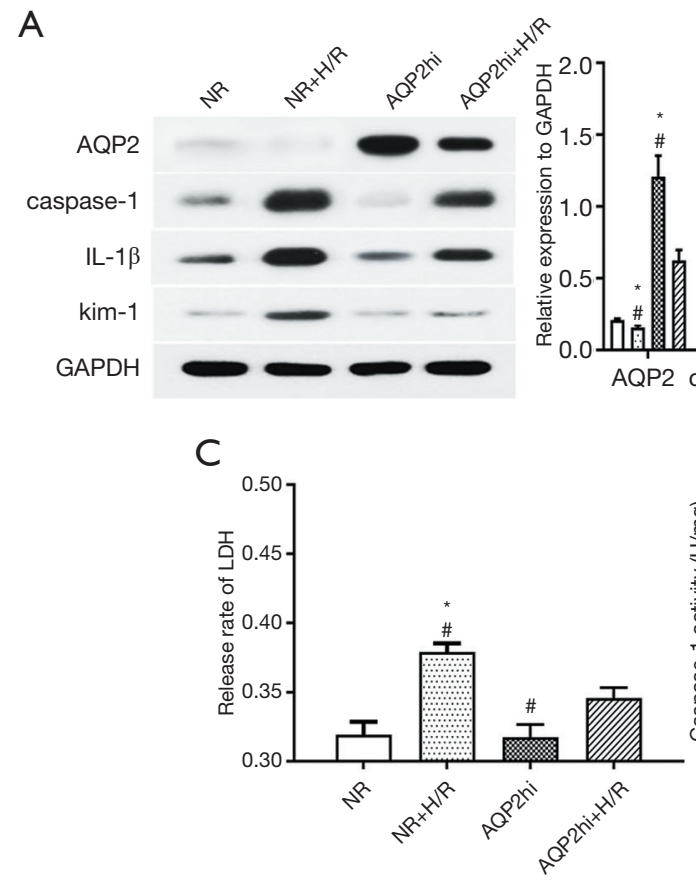
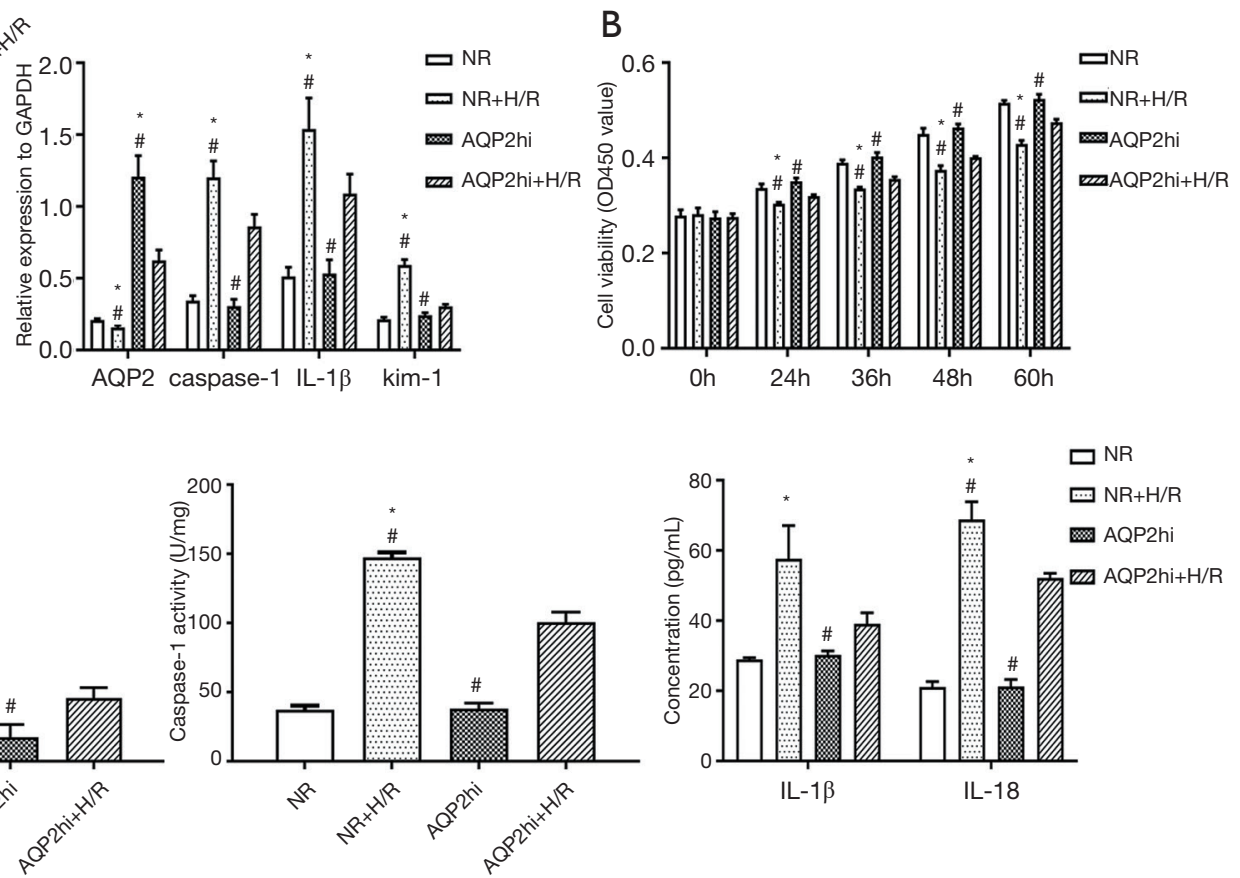

Figure $5 \mathrm{AQP} 2$ overexpression alleviates the pyroptosis of HK-2 cells. (A) Western blot analysis. The cells that overexpressed AQP2, whether treated with H/R (the AQP2hi + H/R group) or not (the AQP2hi group) showed significantly higher AQP2 expression than did the cells created with non-related blank vectors, whether treated with $\mathrm{H} / \mathrm{R}$ (the NR $+\mathrm{H} / \mathrm{R}$ group) or not (the NR group), respectively. The NR $+\mathrm{H} /$ $\mathrm{R}$ and AQP2hi + H/R groups expressed significantly less AQP2 but more kim-1, IL-1 $\beta$ and caspase-1 than did the NR and AQP2hi groups, respectively. The AQP2hi + H/R group expressed less kim-1, IL-1 $\beta$ and caspase-1 than the NR + H/R group. (B) Testing with the Cell Counting Kit-8. The OD values at the same time point were all significantly lower in the NR + H/R group than in either the NR or AQP2hi + H/R group after 24-, 36-, 48-, and 60-hour culture. After 24-, 36-, 48-, and 60-hour culture, the OD values of the AQP2hi group were higher than those of the AQP2hi + H/R group but comparable with those of the NR group. (C) Measurement of concentration of IL-1 $\beta$ and IL-18 in supernatant, and release rate of LDH and activity of caspase-1 in cells. The NR + H/R and AQP2hi + H/R groups had significantly higher caspase- 1 activity, release rates of LDH and supernatant concentrations of IL- $1 \beta$ and IL-18 than did the NR and AQP2hi groups, respectively. The AQP2hi + H/R group had lower caspase-1 activity, release rates of $\mathrm{LDH}$ and supernatant concentrations of IL-1 $(\mathrm{P}=0.065)$ and IL-18 than did the NR + H/R group. * means $\mathrm{P}<0.05$ (compared with the NR group); \# means $\mathrm{P}<0.05$ (compared with the AQP2hi + H/ R group:). AQP, aquaporin; HK-2, human kidney 2; H/R, hypoxia-reoxygenation; IL, interleukin; IRI, ischemia-reperfusion injury; kim-1, kidney injury molecule 1; LDH, lactate dehydrogenase; NC, negative control; NR, non-related; OD, optical density.

Current research has confirmed that during IRI, amounts of radical oxygens were generated, accompanied by pro-inflammatory response, which increased renal cell apoptosis, autophagy and irreversible cell death (13). While, increasing expression of AQPs could reduce cell injury by reducing oxidative stress, promoting metabolism, and inhibiting the signals involving apoptosis and inflammation. We also confirmed that the IRI group in vivo had higher expression of Toll-like receptor 4, caspase-1, kim1, and IL$1 \beta$; higher caspase-1 activity; and higher concentrations of IL- $1 \beta$ and IL-18 in blood; while less AQP2 expression were observed. Similar results were also observed in HK-2 cells in vitro experiments, and the H/R group cells were found to have more pyroptosis by FAM-YVAD-FMK/Hoechst/ PI staining. The overexpression of AQP2 partially reversed the above changes of HK-2 cells. These results revealed the potential modulatory role of AQP2 in pyroptosis in the proximal tubule, and overexpression of AQP2 might reduce injury from renal IRI and improve renal outcome.

Pyroptosis is a type of cell death with different mechanism, characteristics, and outcome from those of apoptosis or necrosis (14). According to reported studies, the process of tissue IRI accompanies the occurrence of pyroptosis and decrease of AQPs levels $(11,15)$, and these 
were consistent with the results we observed in the mice kidneys of IRI model. Caspase-1 is capable of cleaving Gasdermin D, which is essential to promote pyroptosis, to the formation of large pores in the plasma membrane (16). Furthermore, caspase- 1 promote IL- $1 \beta$ and IL-18 from inactive precursors to maturation through these formed pores or upon cell lysis, then leading to pyroptosis (14). Pyroptosis plays an important role in renal IRI (17). In this study, the upregulation of caspase-1, IL- $1 \beta$, and IL-18 were observed in IRI group, suggesting that caspase-1-mediated pyroptosis may be involved in renal IRI. The suppression of pyroptosis potentially prevented the process of organ IRI. Chun et al. found that, in rats, hydrogen sulfide protected against lung injury after limb ischemia-reperfusion by alleviating inflammation and AQP1/AQP5 expression abnormalities (18). Besides, AQP3 deletion in murine kidneys resulted in worse IRI outcomes (8). However, to our knowledge, no other study has focused on the relationship between AQPs and pyroptosis in renal IRI. In this study, the expression of AQP2 in HK-2 cells in the H/R group was significantly down-regulated, accompanied by lower cell proliferation rate, more release of IL- $1 \beta$ and IL-18, and more pyroptosis. However, the overexpression of AQP2 in HK-2 cells exhibited milder pyroptosis and less cell injury than that of the control cells after H/R. The results of this study indicate that increased AQP2 expression in HK-2 cells may reduce pyroptosis during H/R.

The lower expression of AQP2 was observed in IRI groups, accompanied by an increase in pyroptosis, which was consistent with other studies $(19,20)$. Besides, this study showed that the exogenous overexpression of AQP2 attenuated the HK-2 cell damage and pyroptosis caused by $H / R$, which may be related to the down-regulation of caspase- 1 expression and activity. However, this study still had some limitations. On the one hand, we only studied the effects of overexpression of AQP2 on the proliferation and pyroptosis of HK-2 cells, the exact mechanism of AQP2 in modulating pyroptosis and the changes in renal physiological function after AQP2 overexpression remained unknown. On the other hand, it is thought that AQP2 expression is mainly expressed in the distal tubules and collecting ducts. However, some studies suggested that the expression of AQP2 could also be detected in HK-2 cells, suggesting that the weakly expressed AQP2 in renal proximal tubular cells may be a potential research target $(21,22)$. Furthermore, the pathophysiological mechanism of kidney IRI is complex. Increasing the expression of AQP2 alone may not completely reverse the injury of renal tubular cells induced by ischemia-reperfusion, but a potential therapeutic target. Currently, it is reported that calcium and cAMP/PKA signaling activators can induce AQP2 activation. Prostaglandin E2 (PGE2) receptors EP2 and EP4, can selectively increase the transcription and membrane accumulation of AQP2 (23). Moreover, EP4 has been confirmed to be able to attenuates renal ischemiareperfusion (I/R) injury by against I/R-induced oxidative stress, necrosis, apoptosis and autophagy $(24,25)$. In further studies, more in vivo animal experiments will be carried out to explore the effect of overexpression of AQP2 on the kidneys of IRI and its potential mechanism. Due to the large physiological differences between humans and mice, the application of the results of this study to the clinic still needs more researches.

\section{Conclusions}

This study provided a novel insight into the modulatory role of AQP2 in pyroptosis of HK-2 cells. These results indicated that increasing AQP2 expression in proximal tubular cells could potentially reduce pyroptosis during renal IRI. Drugs targeting AQPs may have broad potential clinical applications (26). The development of reagents that increase AQP2 expression for the treatment of renal IRI is expected in the foreseeable future.

\section{Acknowledgments}

Funding: This research was supported by the National Clinical Research Center for Geriatrics, West China Hospital, Sichuan University (Z20201011), National Natural Science Foundation of China (Grant No. 81870513, .81600584, 81470980), A Special Supportive Program for Organ Transplantation by COTDF (Grant no. 2019JYJH08), 1.3.5 project for disciplines of excellence, West China Hospital, Sichuan University (Grant no. ZY2016104), Youth researcher funding of Sichuan University (Grant no.2017SCU11042) and Research funding of Sichuan health and family planning commission (Grant no. 17PJ159, 18PJ434, and 18PJ453).

\section{Footnote}

Reporting Checklist: The authors have completed the ARRIVE reporting checklist. Available at http://dx.doi. org/10.21037/tau-21-71

Conflicts of Interest: All authors have completed the ICMJE 
uniform disclosure form (available at http://dx.doi. org/10.21037/tau-21-71). The authors have no conflicts of interest to declare.

Ethical Statement: The authors are accountable for all aspects of the work in ensuring that questions related to the accuracy or integrity of any part of the work are appropriately investigated and resolved. The Animal Ethics Committee of West China Hospital, Sichuan University, Chengdu, China (No. 2019141A) approved this study. All experimental procedures were followed by the National Institutes of Health Guide for the Care and Use of Laboratory Animals.

Open Access Statement: This is an Open Access article distributed in accordance with the Creative Commons Attribution-NonCommercial-NoDerivs 4.0 International License (CC BY-NC-ND 4.0), which permits the noncommercial replication and distribution of the article with the strict proviso that no changes or edits are made and the original work is properly cited (including links to both the formal publication through the relevant DOI and the license). See: https://creativecommons.org/licenses/by-nc-nd/4.0/.

\section{References}

1. Holmberg C, Jalanko H. Long-term effects of paediatric kidney transplantation. Nat Rev Nephrol 2016;12:301-11.

2. Wong G, Teixeira-Pinto A, Chapman JR, et al. The impact of total ischemic time, donor age and the pathway of donor death on graft outcomes after deceased donor kidney transplantation. Transplantation 2017;101:1152-8.

3. Böhmová R, Viklický O. Renal ischemia--reperfusion injury: an inescapable event affecting kidney transplantation outcome. Folia Microbiol (Praha) 2001;46:267-76.

4. Veighey KV, Nicholas JM, Clayton T, et al. Early remote ischaemic preconditioning leads to sustained improvement in allograft function after live donor kidney transplantation: long-term outcomes in the REnal Protection Against Ischaemia-Reperfusion in transplantation (REPAIR) randomised trial. Br J Anaesth 2019;123:584-91.

5. Rabolli V, Wallemme L, Lo Re S, et al. Critical role of aquaporins in interleukin $1 \beta$ (IL-1 $\beta$ )-induced inflammation. J Biol Chem 2014;289:13937-47.

6. Törnroth-Horsefield S. Phosphorylation of human AQP2 and its role in trafficking. Vitam Horm 2020;112:95-117.

7. Ayasoufi K, Kohei N, Nicosia M, et al. Aquaporin 4 blockade improves survival of murine heart allografts subjected to prolonged cold ischemia. Am J Transplant 2018;18:1238-46.

8. Lei L, Wang W, Jia Y, et al. Aquaporin-3 deletion in mice results in renal collecting duct abnormalities and worsens ischemia-reperfusion injury. Biochim Biophys Acta Mol Basis Dis 2017;1863:1231-41.

9. HK-2 (ATCC@ CRL-2190 ${ }^{\mathrm{TM}}$ ) Available online: https://www.atcc.org/products/all/ CRL-2190. aspx\#culturemethod.

10. Tang TT, Lv LL, Pan MM, et al. Hydroxychloroquine attenuates renal ischemia/reperfusion injury by inhibiting cathepsin mediated NLRP3 inflammasome activation. Cell Death Dis 2018;9:351.

11. Asvapromtada S, Sonoda H, Kinouchi M, et al. Characterization of urinary exosomal release of aquaporin-1 and -2 after renal ischemia-reperfusion in rats. Am J Physiol Renal Physiol 2018;314:F584-601.

12. Klein K, Rübenacker S, Schaefer SM, et al. Tissue expression of aquaporin 2 is correlated to urine output and allograft function in sensitized kidney transplant patients. Transplant Proc 2016;48:2629-36.

13. Weight SC, Bell PR, Nicholson ML. Renal ischaemia-reperfusion injury. Br J Surg 1996;83:162-70.

14. Green DR. The Coming Decade of Cell Death Research: Five Riddles. Cell 2019;177:1094-107.

15. Kristensen ML, Kierulf-Lassen C, Nielsen PM, et al. Remote ischemic perconditioning attenuates ischemia/ reperfusion-induced downregulation of AQP2 in rat kidney. Physiol Rep 2016;4:e12865.

16. Shi J, Gao W, Shao F. Pyroptosis: Gasdermin-Mediated Programmed Necrotic Cell Death. Trends Biochem Sci 2017;42:245-54.

17. Bergsbaken T, Fink SL, Cookson BT. Pyroptosis: host cell death and inflammation. Nat Rev Microbiol 2009;7:99-109.

18. An P, Xie J, Qiu S, et al. Hispidulin exhibits neuroprotective activities against cerebral ischemia reperfusion injury through suppressing NLRP3-mediated pyroptosis. Life Sci 2019;232:116599.

19. Lee S, Kim W, Moon SO, et al. Rosiglitazone ameliorates cisplatin-induced renal injury in mice. Nephrol Dial Transplant 2006;21:2096-105.

20. Suh SH, Lee KE, Kim IJ, et al. Alpha-lipoic acid attenuates lipopolysaccharide-induced kidney injury. Clin Exp Nephrol 2015;19:82-91.

21. Yu G, Liu Q, Dong X, et al. Inhibition of inflammation using diacerein markedly improved renal function in 
endotoxemic acute kidney injured mice. Cell Mol Biol Lett 2018;16;23:38.

22. Barranco R, Ventura F, Fracasso T. Immunohistochemical renal expression of aquaporin 2, arginine-vasopressin, vasopressin receptor 2 , and renin in saltwater drowning and freshwater drowning. Int J Legal Med 2020;134:1733-40.

23. Olesen ET, Rützler MR, Moeller HB, et al. Vasopressinindependent targeting of aquaporin-2 by selective E-prostanoid receptor agonists alleviates nephrogenic diabetes insipidus. Proc Natl Acad Sci U S A 2011;108:12949-54.

Cite this article as: Fan Y, Ma M, Feng X, Song T, Wei Q, Lin T. Overexpression of aquaporin 2 in renal tubular epithelial cells alleviates pyroptosis. Transl Androl Urol 2021;10(6):23402350. doi: 10.21037/tau-21-71
24. Yang CC, Chen KH, Hsu SP, et al. Augmented renal prostacyclin by intrarenal bicistronic cyclo-oxygenase-1/ prostacyclin synthase gene transfer attenuates renal ischemia-reperfusion injury. Transplantation 2013;96:1043-51.

25. Ding C, Han F, Xiang H, et al. Role of prostaglandin E2 receptor 4 in the modulation of apoptosis and mitophagy during ischemia/reperfusion injury in the kidney. Mol Med Rep 2019;20:3337-46.

26. Tradtrantip L, Jin BJ, Yao X, et al. Aquaporin-targeted therapeutics: state-of-the-field. Adv Exp Med Biol 2017;969:239-50. 


\section{Supplementary}

Primers included (from $5^{\prime}$ to $\left.3^{\prime}\right)$ :

h-AQP1 (194 bp): F- ATTTTCTGGGTGGGGCCATT, R- GGGCCAGACCCCTTCTATTT;

m-AQP1 (124 bp): F- AGGCTTCAATTACCCACTGGA, R- GTGAGCACCGCTGATGTGA;

h-AQP2 (137 bp): F- CAGTTCCTGGCATCTCTTGT, R- CCTTTGTCCTTCCCTAACCTATC;

m-AQP2 (137 bp): F- ATGTGGGAACTCCGGTCCATA, R- ACGGCAATCTGGAGCACAG;

h-AQP3 (107 bp): F- ACCCCTCTGGACACTTGGAT, R- GGGGTTGTTGTAGGGGTCAA; m-AQP3 (128 bp): F- GCTTTTGGCTTCGCTGTCAC, R- TAGATGGGCAGCTTGATCCAG;

h-AQP4 (124bp): F- TGGTGCCAGCATGAATCCC, R- CTCATAAAGGCCACCAGCGA;

m-AQP4 (199 bp): F- AGTCACCACGGTTCATGGAAA, R- CATGCTGGCTCCAGTATAATTGA;

h-AQP5 (224 bp): F- CACGTATGAGCCTGACGAGG, R- CCCCTCTCTAACTGTGCAGC; m-AQP5 (220 bp): F- AGAAGGAGGTGTGTTCAGTTGC, R- GCCAGAGTAATGGCCGGAT;

h-AQP8 (126 bp): F- TGCCTGTCGGTCATTGAGAA, R- CGCAGGGTTGAAGTGTCCA; m-AQP8 (145 bp): F- TGTGTAGTATGGACCTACCTGAG, R- ACCGATAGACATCCGATGAAGAT; 


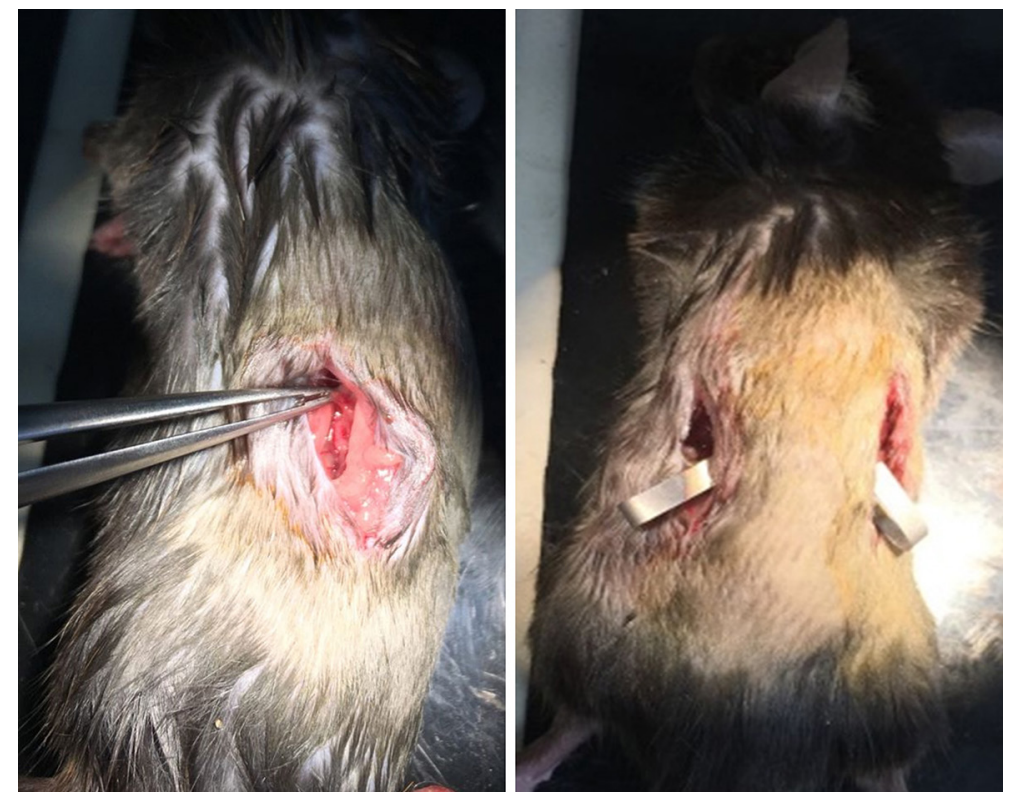

Figure S1 Establishment of renal IRI model. Proper anesthesia was achieved by intra-peritoneal injection of pentobarbital into mice. Both kidneys were exposed along the dorsal incision. After being dissociated, both renal pedicles were clamped with clips simultaneously for 30 minutes. The clips were then removed, and the wound was sutured. IRI, ischemia-reperfusion injury.

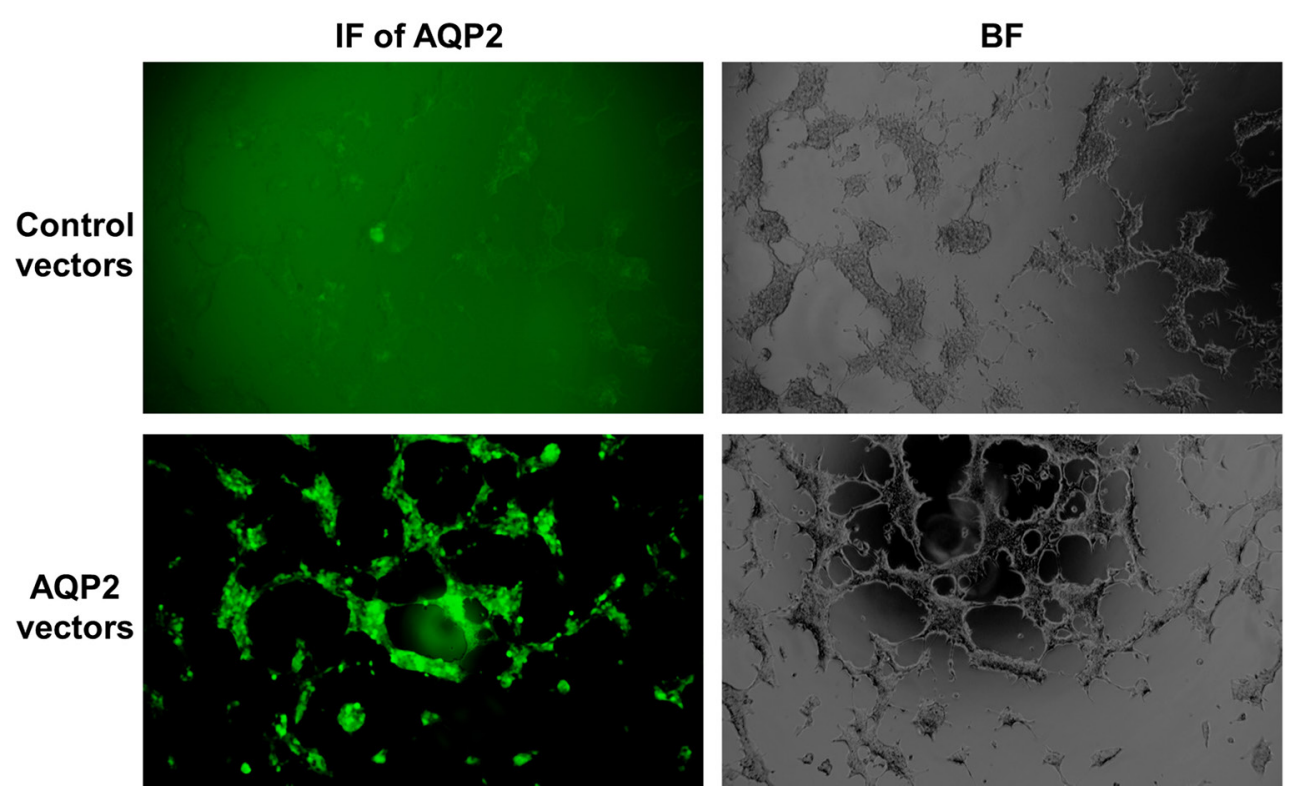

Figure S2 Measurement of AQP2 vectors. The titres of the AQP2 and control vectors were $2.2 \times 10^{5} \mathrm{tu} / \mathrm{mL}$ and $2 \times 10^{5} \mathrm{tu} / \mathrm{mL}$, respectively. $\mathrm{AQP}$, aquaporin. 